\title{
EDITORIAL: Special Issue of 2018 India International Congress on Computational Intelligence
}

\author{
Suash $\mathrm{Deb}^{1,2} \cdot \mathrm{Ka}$-Chun Wong ${ }^{3} \cdot \mathrm{Xin}^{-S h e} \mathrm{Yang}^{4}$ \\ Published online: 7 August 2020 \\ (C) Springer-Verlag London Ltd., part of Springer Nature 2020
}

Dear readers,

Welcome to this special issue of NCAA journal consisting of a collection of four extended papers from ISCMI18 (2018 5th International Conference on Soft Computing \& Machine Intelligence) and ISMSI18 (2018 2nd International Conference on Intelligent Systems, Metaheuristics \& Swarm Intelligence), the 2 annual global events of India International Congress on Computational Intelligence (IICCI) as were held in the year 2018 in Nairobi, Kenya and Phuket Island, Thailand respectively. These 2 conferences endeavor to offer an effective scientific platform for discussing \& disseminating the findings of the latest research efforts in the fields of soft computing $\&$ the related areas of intelligent systems.

The four papers for this special issue were selected from among all the accepted manuscripts on completion of a rigorous reviewing procedure. At first, a subset of the conference papers was shortlisted by the guest editors. The authors of those papers were communicated and asked to extend their respective manuscripts by adding at least, $50 \%$ additional contents. On submission, each of such revised manuscripts had to undergo scrutiny by the team of guest editors and, after being satisfied, was sent to at least 5 independent reviewers, in accordance with the customary reviewing procedures of the journal. Final decisions pertaining to acceptance/rejection of any of those papers were carried out based on the feedbacks provided by the reviewers on completion of the entire reviewing process.

Suash Deb

suash_deb@ieee.org

1 IT and Educational Consultant, Ranchi, Jharkhand, India

2 Decision Sciences and Modelling Program, Victoria University, Melbourne, Australia

3 City University of Hong Kong, Kowloon Tong, Hong Kong

4 Middlesex University, London NW4 4BT, UK
This special issue is aimed at practitioners, researchers and postgraduate students who are engaged in developing and applying advanced intelligent systems principles to solve real-world problems. The intention lies in further strengthening and presenting the state-of-the-art of rapidly evolving areas of Computational Intelligence and some of its components like Swarm Intelligence etc. based on the relevance of the journal. The papers of the same are organized as follows:

The study reported by Yunhe Wang et al. introduced a hypervolume-based discrete evolutionary optimization algorithm (HYBDEOA). It aims at differentiating various subtypes of gene expression data. For ensuring proper classification four parameters namely the number of genes, the accuracy, relevance and the redundancy were optimized in parallel. The robustness of classification of HYBDEOA had been clearly validated by the experimental results.

The one communicated by Jan Kozak et al. proposed a hybrid ant colony optimization and ensemble method for addressing the practical issues in E-mail foldering. In particular, the authors have focused on the stability of the proposed algorithm by incorporating its streaming decision policy into the pheromone of the ant colony optimization algorithm. The proposed approach has been validated and compared with other methods on real-world data, demonstrating its practical uses.

The manuscript of Peter Olukanmi et al. gave us a refreshing view on improving the well-grounded k-means clustering method at the era of big data. The authors have introduced us a highly efficient k-means clustering approach which can be scalable to be run on massive datasets with constant time complexities (namely, k-means-lite). The benchmark results on extensive datasets demonstrated that the performance of k-means-lite and its variants can be comparable to the existing k-means methods with a constant-time behavior. 
The research efforts as reported by Kun Lan et al. consists of introduction of a multi-view convolutional neural network for heart disease and breast cancer detection. In particular, the proposed multi-view convolutional neural network has been carefully designed with particle swarm optimization to achieve accurate target recognition in medical images. The experimental results reflect that the proposed model can gives us encouraging performance when comparing to the existing methods.

We hope you will find this special issue enjoyable, informative and useful.

We take this opportunity of expressing our profound gratitude to all the reviewers, who despite being so busy, had agreed to invest their valued time for examining the contents of the allotted papers and sharing their findings within the stipulated time. Papers passed through various stages of the process of reviewing and the very useful technical inputs, as provided by all of them during each of those steps had definitely helped, to a great extent, to enhance the quality of the manuscripts and in turn, the standing of this special issue. Our thanks go to all the authors who had expanded their respective conference manuscripts and showed remarkable patience throughout the lengthy reviewing process. Last but certainly not the least, we must acknowledge and extend our deep sense of indebtedness to Prof. John MacIntyre, the editor-in-chief of this journal, for his support and guidance during the entire journey of this special issue. 